\title{
An Application of Multi-Face Recognition Based on Feature Extraction
}

\author{
Dongqin $\mathrm{Gu}^{\mathrm{a}}{ }^{*}$, Seiichi Serikawa ${ }^{\mathrm{b}}$, Lifeng Zhang ${ }^{\mathrm{b}}$ \\ a Yangzhou University, Yangzhou, 225000, China \\ ${ }^{\mathrm{b}}$ Kyushu Institute of Technology, Kitakyushu, 8048550, Japan \\ *Corresponding Author: gu.dongqin580@mail.kyutech.jp
}

\begin{abstract}
In order to help people with difficulties in face recognition, we propose an application for multi-face recognition. This paper mainly introduces the application of face detection and recognition algorithm based on feature extraction. Firstly, use smart glasses to get a video. Secondly, the feature extraction algorithm is used for face detection to obtain our own face database and feature file. Finally, face detection and recognition is performed based on the obtained new video. The names of the recognized faces or related information can be displayed, which is used to remind the wearer of the glasses when necessary.
\end{abstract}

keywords: feature extraction, face detection, face recognition.

\section{Introduction}

Recently, the United Nations issued a report that by 2050 , the number of elderly people over the age of 65 in the world will exceed 1.5 billion, accounting for $16 \%$ of the total population. (II) As the aging process intensifies, the number of people with memory declines is increasing. A friend I met yesterday, maybe I can't remember his name today. On the other hand, for those who have face blindness, also known as "face amnesia", others' faces are vague in their eyes, and they have no ability to recognize facial features. They are very poor in distinguishing people's faces, and they can only identify the person through some other characteristics, such as whether to wear glasses, the color of clothes, and so on. The decline or lack of face recognition ability brings a lot of problems. Face recognition plays an important role in daily life.

We use smart glasses to capture a video, and then use the feature extraction algorithm for face detection to get the face database and feature files. Then, face recognition is performed based on the video. Remind the wearer of the glasses that the names of the persons in the video or related information if necessary.

Smart glasses is a pair of glasses that can be connected to a mobile phone or computer to share live videos. We can use it to get videos. The algorithm based on feature extraction includes three parts: face detection, feature extraction and face recognition.

Smart glasses is a pair of glasses that can be connected to a mobile phone or computer to share live videos. We can use it to get videos. The algorithm based on feature extraction includes three parts: face detection, feature extraction and face recognition.

The face detection part uses the histogram of oriented gradient (HOG) and regression tree method. HOG is a description operator that can detect objects based on shape edge features. ${ }^{(\mathbb{L})-(\mathbb{D})}$ It can reflect the edge information of the image target well by utilizing the gradient information. Therefore, the appearance and shape of the image portion can be characterized by the size of the local gradient.

The feature extraction part detects 68 feature points of the face, analyzes the geometric relationship between the feature points, and saves the picture as a 128-dimensional vector by using the deep residual network model.

The face recognition part utilizes a method based on a deep residual network. ${ }^{(6)}$ Using the trained model, the face vector to be tested is calculated and compared with the feature vectors in the feature file we have obtained before. If the minimum Euclidean distance meets the specific conditions, the match is successful. 


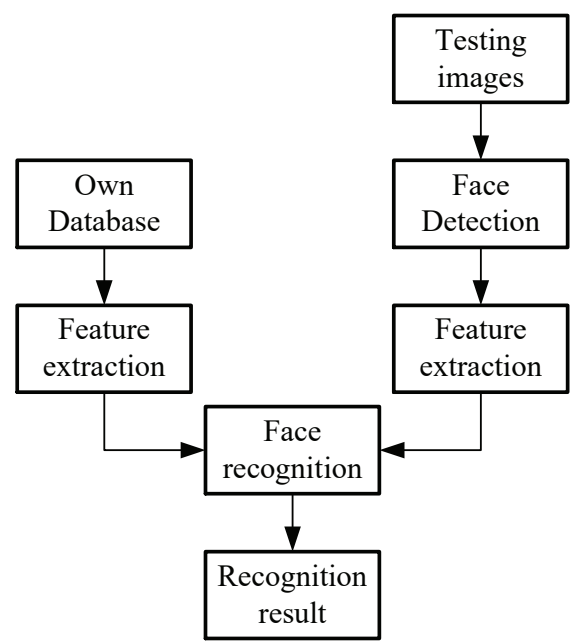

Fig. 1. Face recognition system

\section{Related work}

As shown in Fig. [, the face recognition system mainly includes the following parts: face detection, face database construction, feature extraction and face recognition. In the face database construction section, a single face image set is constructed by face detection after selecting a video. Organize and classify to obtain a face database. In the feature extraction section, face information is extracted and a face feature document is generated. The test face is matched with the information in the feature document, and display the recognition result in the face recognition section.

\subsection{Face Detection}

We use the HOG and regression tree methods in Dlib. ${ }^{(\mathbb{D})-(\mathbb{( \Phi )}}$ The HOG constitutes a feature by calculating and counting a gradient direction histogram of a local region of the image. And the workflow is shown in Fig. [.

On the other hand, Dlib is a C++ open source toolkit that includes machine learning algorithms. Using the Dlib-trained model, the frontal face detector, which used the principle of HOG in the Dlib library is called for face detection. The specific operation is as follows. Firstly, read the test picture and convert it to grayscale. Secondly, use Dlib frontal face detector to detect if the picture contains a human face. Thirdly, if there is a face, the face is framed, otherwise the next picture is detected.

There are also some other methods such as face detection using Haar cascades. Haar features are divided into three categories: edge features, linear features, center features, and diagonal features, combined into feature templates. The feature template has two rectangles, white and black, and defines the template's feature values as the sum of the white

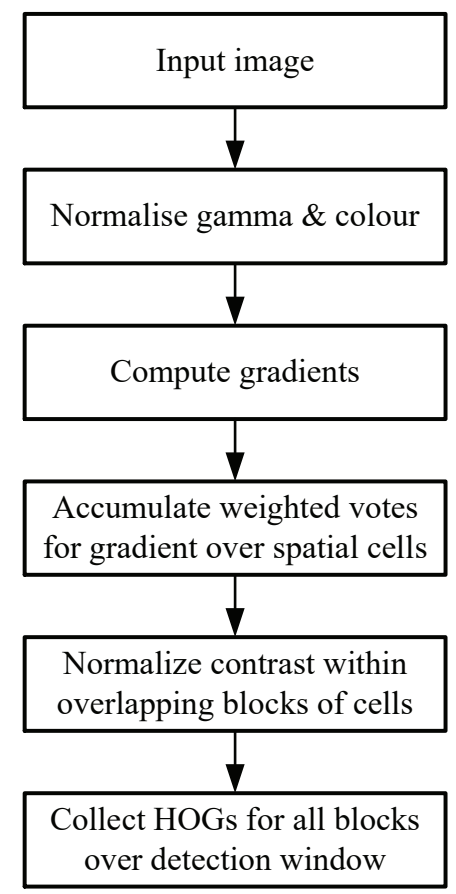

Fig. 2. Workflow of the HOG

Table 1. Correct rate of face detection with different methods.

\begin{tabular}{|c|c|c|}
\hline Method & Test Faces & Correct Rate \\
\hline Haar & 168 & $69.64 \%$ \\
\hline Dlib & 168 & $97.62 \%$ \\
\hline
\end{tabular}

rectangle pixels minus the sum of the black rectangle pixels. Haar eigenvalue reflects the grayscale variation of the image. For example, some features of the face can be described simply by the rectangular features, such as: the eyes are darker than the cheeks, the sides of the bridge are darker than the nose, and the mouth is darker than the surrounding.

However, Haar cascades method has a poor face detection effect. Faces with positive, vertical and good rays of light can be detected by this method, while faces with side, skew and bad rays of light cannot be detected.

We have detected the same group of test faces by using Haar cascades and Dlib. The correct rate of face detection with two methods can be show as Table $\mathbb{\text { I. }}$. We can see that face detection using Dlib is better than Haar cascades. Therefore, we chose the Dlib method for face detection.

\subsection{Face Database Construction}

As shown in Fig. B, a video is acquired through the smart glasses. Then start the face detection after converting the video to pictures. If a face is detected, the face image is saved and used to construct our own face database, otherwise the image is not saved. 


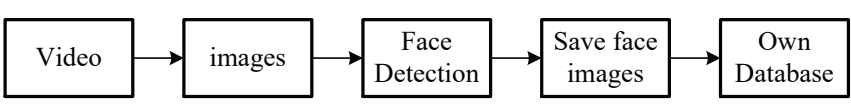

Fig. 3. Face database construction process

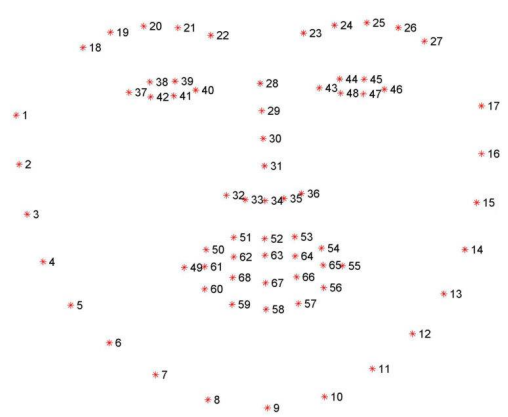

Fig. 4. 68 feature points of the face

\subsection{Feature Extraction}

In the feature extraction section, a given image is processed, and facial features are identified and extracted.

As shown in Fig. 田, the eyes, nose, mouth and overall facial contour are primarily marked. Calculate the geometric relationship between these points and convert the face image into a form of feature vector, and save it to a feature file.

We use the 68 feature points ${ }^{(\Phi)}$ of the face in the Dlib library. The model constructed with the depth residual network saves the picture in the form of a 128-dimensional vector. Since a person has multiple photos, each person's feature mean is calculated and saved into a feature file of the csv type.

\subsection{Face Recognition}

The camera is read in real time, and the face feature vector is obtained by using the deep residual network model in Dlib. Calculate the Euclidean distance of the feature vector of the test face and each feature vector in the database. If the minimum Euclidean distance meets certain threshold conditions, the match is successful, and the name is displayed below the face frame, otherwise display unknown.

\section{Experiment Results}

\subsection{Hardware and Software}

In order to realize the face recognition function of the smart glasses wearer, the required hardware and software are as follows.

Hardware: Wi-Fi Live Glasses, as shown in Fig. [.

Software: Anaconda environment, python 3.5, OpenCV 3.4.2; installation libraries: numpy $==1.15 .0$, Dlib $==19.17 .0$ and scikit-image $==0.14 .0$.

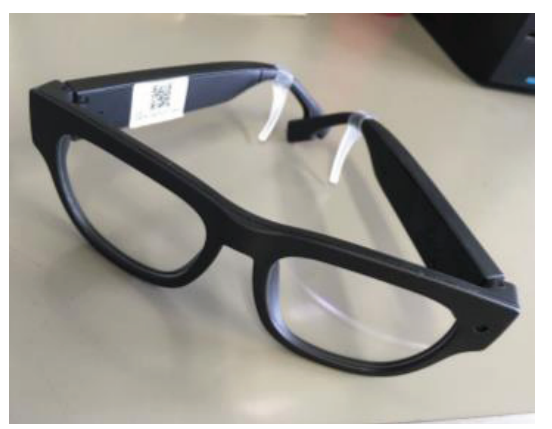

Fig. 5. Wi-Fi Live Glasses

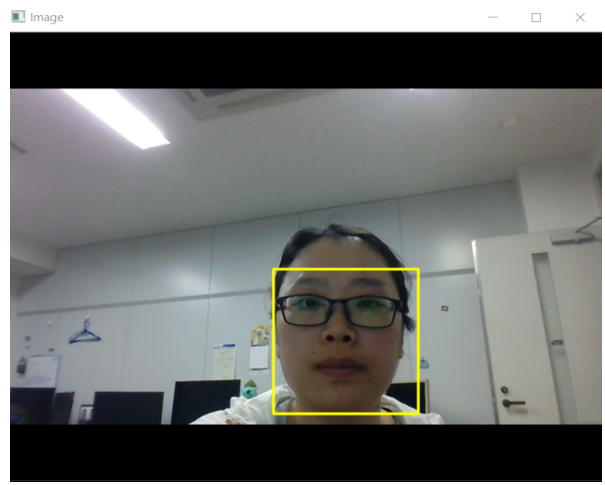

Fig. 6. Face detection

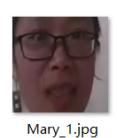

Mary 1.jpg
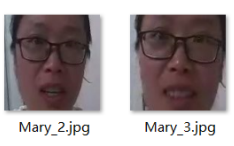
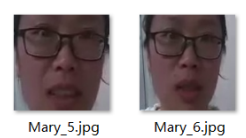

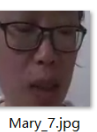

Mary_.jpg
Fig. 7. Single face

\subsection{Face Database}

This experiment uses Wi-Fi Live Glasses to capture a video with multiple faces, and through face detection and processing to convert the video into multiple faces with the same size. Pictures of different people are stored in different folders and the folders are named in the form of "person" + "_" +number. The same person can have multiple face photos for feature extraction. To create a face database, we used Wi-Fi Live Glasses for video capture to simulate the video taken by the glasses wearer.

In this experiment, the faces of 30 people appearing in the video were extracted. The face detection, the single face picture after the picture processing, and the constructed face database are shown in Fig. ฤ, Fig. \and Fig. §, respectively.

Get 68 feature points of each picture in a folder from the face database. Convert all the faces into corresponding 128dimensional vectors and then calculate the person's feature mean. Save to a csv file. Traverse all folders to form a face feature file for face matching. There are 30 people in the 


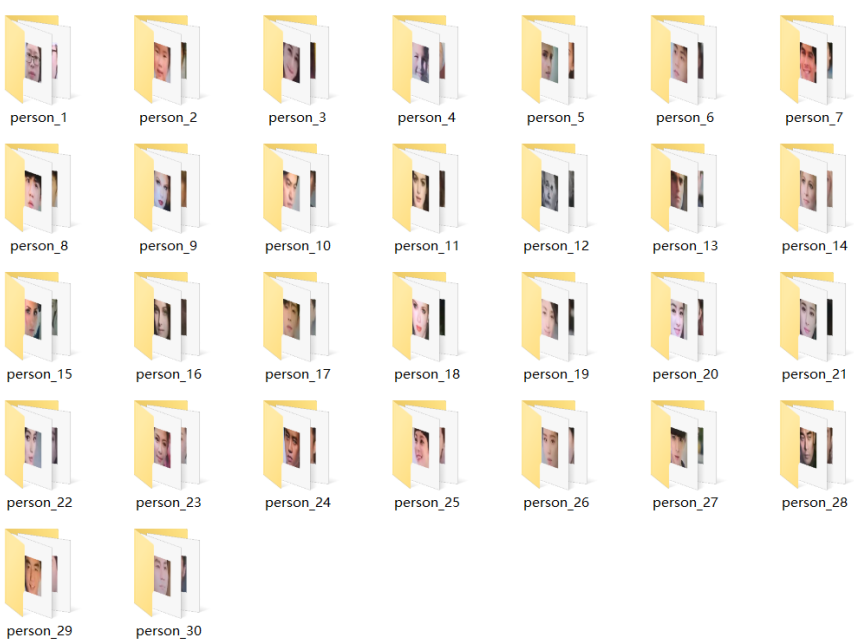

Fig. 8. My own Database

face database in this experiment. Therefore, there are 30 128-dimensional feature vectors in the csv file.

\subsection{Face Recognition}

Read the video and check if the video contains a human face. If there is a face, extract the face features in the video and save the face as a 128-dimensional vector. Calculate the Euclidean distance between the vector of the test face and all feature vectors in the csv file.

The Euclidean distance of two n-dimensional space vectors $x$ and $y$ is defined as

$$
D(x, y)=\sqrt{\sum_{k=1}^{n}\left(x_{k}-y_{k}\right)^{2}} .
$$

Assuming that there are $\mathrm{N}$ people's face data in the database, a csv file containing N 128-dimensional vectors can be obtained. Calculate the Euclidean distance between the test face vector and each vector in csv, we can obtain that

$$
D_{i}=D_{i_{1}}, D_{i_{2}}, \cdots, D_{i_{N}}
$$

This experiment has 30 people in the face database, so we can get 30 Euclidean distances, i.e., $D_{1}, D_{2}, \cdots, D_{30}$. Compare these Euclidean distances, find the minimum one.

$$
D=\min \left\{D_{1}, D_{2}, \cdots, D_{30}\right\}
$$

If Eq. (4) is satisfied, the match is successful, and the name of the corresponding person is output, otherwise unknown is displayed.

$$
D \leq \text { Threshold, }
$$

where the Threshold is a constant.
Table 2. Correct rate of face recognition under different thresholds.

\begin{tabular}{|c|c|c|}
\hline Threshold & Test Faces & Correct Rate \\
\hline 0.35 & 134 & $68.70 \%$ \\
\hline 0.4 & 134 & $89.31 \%$ \\
\hline 0.45 & 134 & $94.66 \%$ \\
\hline 0.5 & 134 & $96.95 \%$ \\
\hline 0.55 & 134 & $95.42 \%$ \\
\hline 0.6 & 134 & $91.60 \%$ \\
\hline
\end{tabular}

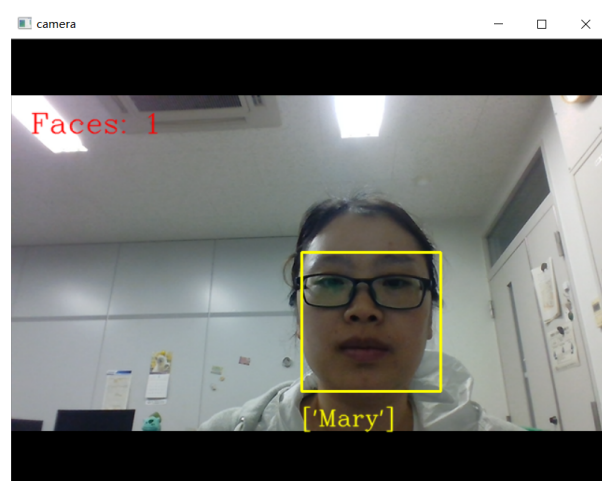

Fig. 9. Single face recognition result

We experimented with different thresholds using our own face database. Select 70 pictures containing 134 faces for face detection and recognition. The experimental results are shown in Table [.

As shown in Table 2 , different thresholds have different correct rate of face recognition. It should be set according to the actual situation. For security-critical situations, such as security, a small threshold is required to ensure safety. When the suspect is tracking, a larger threshold is needed to ensure that the suspect is not missed.

The face recognition results are shown in Fig. 9 and Fig. [0]. In the upper left corner of the Figs, the number of faces detected is displayed. Fig. 9 shows the single face recognition result. Fig. 10 shows the multiple face recognition result. The faces to be tested in Fig. 10(a) are all in the database, and the corresponding names are displayed respectively. In Fig. 10 (b), one face is not in the database and it is displayed as unknown.

\section{Conclusions and Future Work}

This paper designed a face recognition algorithm based on feature extraction. Used Wi-Fi Live Glasses as a medium to help the wearer remember the information about people in the video. Of course, you can also choose a mobile phone or we- 


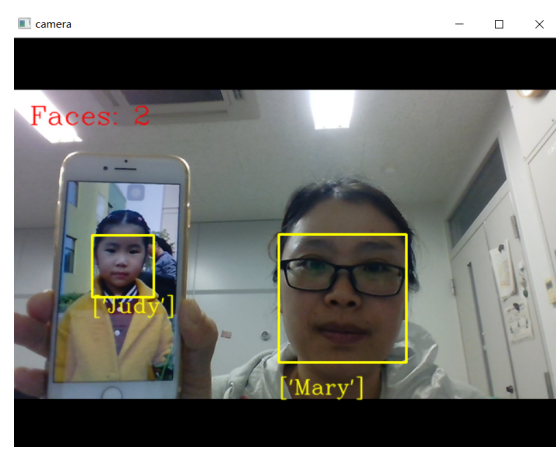

(a) Both two faces are all in the database.

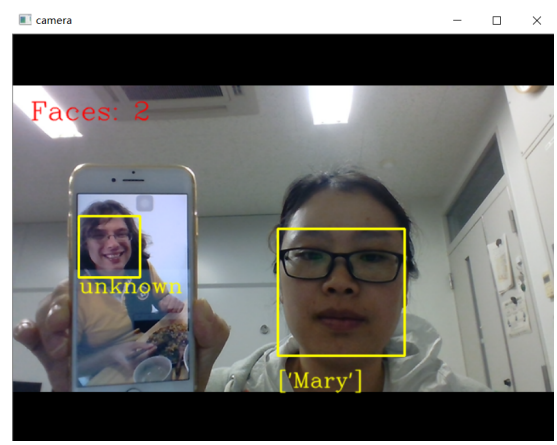

(b) One face is not in the database.

Fig. 10. Multiple faces recognition results

bcam to get the video. The experimental results showed that this method could correctly detect and recognize faces in the video. However, the algorithm also has some shortcomings. Firstly, when the light is insufficient, the face we captured is not clear enough, and the correctness of face recognition will be affected. Secondly, using Wi-Fi Live Glasses to watch a video on the network, the quality of image is not high, and sometimes the faces can't be detected. The future work is to solve these problems by improving the algorithm.

\section{Acknowledgment}

This work was supported by Yangzhou University International Academic Exchange Fund.

\section{References}

(1) UN report: the proportion of the elderly population will reach $1 / 6$ in 2050, https://cn.nikkei.com/ politicsaeconomy/politicsa-society/350002019-04-03-08-59-28.htmL, Nikkei Chinese, Accessed: 2019.5.18

(2) A. Ade-Ibijola, and K. Aruleba : "Automatic Attendance Capturing Using Histogram of Oriented Gradients on Facial Images", 2018 IST-Africa Week Conference (IST-Africa), 1-8, 2018
(3) G. Tsai : "Histogram of oriented gradients," University of Michigan, 2010

(4) N. Dalal, and B. Triggs : "Histograms of oriented gradients for human detection”, CVPR, 2005

(5) T. Surasak, I. Takahiro, C. Cheng, C. Wang, and P. Sheng : "Histogram of oriented gradients for human detection in video", 2018 5th International Conference on Business and Industrial Research (ICBIR), 172-176, 2018

(6) K. He, X. Zhang, S. Ren, and J. Sun : "Deep Residual Learning for Image Recognition”, 2016 IEEE Conference on Computer Vision and Pattern Recognition (CVPR), 770-778, 2016

(7) Dlib Python API Tutorials [Electronic resource] - Access mode : http://dlib.net/python/index.html

(8) X. Ren, J. Ding, J. Sun, and Q. Sui : "Face modeling process based on Dlib", 2017 Chinese Automation Congress (CAC), 1969-1972, 2017

(9) N. Boyko, O. Basystiuk, and N. Shakhovska : "Performance Evaluation and Comparison of Software for Face Recognition, Based on Dlib and Opencv Library”, 2018 IEEE Second International Conference on Data Stream Mining Processing (DSMP), 478-482, 2018 